\title{
Multiepitope $\mathrm{CD8}^{+} \mathrm{T}$ cell response to an NY-ESO-1 peptide vaccine results in imprecise tumor targeting
}

\author{
Valérie Dutoit, ${ }^{1,2}$ Robert N. Taub, ${ }^{3}$ Kyriakos P. Papadopoulos, ${ }^{3}$ Susan Talbot, ${ }^{1}$ \\ Mary-Louise Keohan, ${ }^{3}$ Michelle Brehm, ${ }^{1}$ Sacha Gnjatic, ${ }^{4}$ Paul E. Harris, ${ }^{3}$ \\ Brygida Bisikirska, ${ }^{3}$ Philippe Guillaume, ${ }^{5}$ Jean-Charles Cerottini, ${ }^{5}$ \\ Charles S. Hesdorffer, ${ }^{3}$ Lloyd J. Old, ${ }^{4}$ and Danila Valmori ${ }^{1,2}$
}

${ }^{1}$ Ludwig Institute Clinical Trial Center, New York Branch at Division of Medical Oncology, Department of Medicine, Columbia University College of Physicians and Surgeons, New York, New York, USA

${ }^{2}$ Division of Clinical Onco-Immunology, Ludwig Institute for Cancer Research, University Hospital, Lausanne, Switzerland

${ }^{3}$ Division of Medical Oncology, Department of Medicine, Columbia University College of Physicians and Surgeons,

New York, New York, USA

${ }^{4}$ Ludwig Institute for Cancer Research, New York Branch at Memorial Sloan-Kettering Cancer Center,

New York, New York, USA

${ }^{5}$ Ludwig Institute for Cancer Research, Lausanne Branch, University of Lausanne, Epalinges, Switzerland

\begin{abstract}
The cancer-testis antigen NY-ESO- 1 is one of the most promising candidates for generic vaccination of cancer patients. Here we analyzed the $\mathrm{CD}^{+} \mathrm{T}$ cell response to a NY-ESO-1 peptide vaccine composed of the two previously defined peptides 157-165 and 157-167, administered with GM-CSF as a systemic adjuvant. The NY-ESO-1 peptide vaccine elicited a $\mathrm{CD8}^{+} \mathrm{T}$ cell response directed against multiple distinct epitopes in the 157-167 region, as revealed by using A2/peptide multimers incorporating overlapping A2 binding peptides in this region. However, only a minor fraction of the elicited $\mathrm{CD8}^{+} \mathrm{T}$ cells, namely those recognizing the peptide $157-165$ with sufficiently high functional avidity, recognized the naturally processed target on NY-ESO- $1^{+}$tumor cells. In contrast, the majority of peptide 157-165-specific $\mathrm{CD8}^{+} \mathrm{T}$ cells exhibited lower functional avidity and no tumor reactivity. In addition, vaccine-elicited $\mathrm{CD8}^{+} \mathrm{T}$ cells specific for other overlapping epitopes in the 157-167 region failed to significantly recognize NY-ESO-1-expressing tumor targets. Thus, because of the complexity of the $\mathrm{CD8}^{+} \mathrm{T}$ cell repertoire that can be elicited by vaccination with synthetic peptides, a precise definition of the targeted epitope, and hence, of the corresponding peptide to be used as immunogen, is required to ensure a precise tumor targeting.
\end{abstract}

J. Clin. Invest. 110:1813-1822 (2002). doi:10.1172/JCI200216428.

\section{Introduction}

In recent years, the analysis of spontaneous immune responses to autologous tumors in cancer patients has allowed the identification of several categories of tumor-associated antigens that can be the target of more or less tumor-specific immune responses (1-4). Among them, one of the most relevant for the development of cancer vaccines is the group of the so-called

Received for publication July 15, 2002, and accepted in revised form October 18, 2002.

Address correspondence to: Danila Valmori, Ludwig Institute Clinical Trial Center, New York Branch at Division of Medical Oncology, Department of Medicine, Columbia University College of Physicians and Surgeons, 650 West 168th Street, Black Building Room 20-22, New York, New York 10032, USA. Phone: (212) 305-3923; Fax: (212) 305-6798;

E-mail: danila.valmori@inst.hospvd.ch.

Conflict of interest: The authors have declared that no conflict of interest exists.

Nonstandard abbreviations used: cancer-testis (CT); enzymelinked immunosorbent spot (ELISPOT); Tris [2-carboxyethyl] phosphine (TCEP); effector-to-target cell ratio (E/T); A2/peptide multimers $(\mathrm{M})$. cancer-testis (CT) antigens, which are expressed by tumor cells but not by most somatic adult tissues, with the exception of testis, for example, MAGE (5) and NY-ESO-1 (6). Because their expression is commonly found in tumors of various histological types, $\mathrm{CT}$ antigens are the main candidates for generic vaccination of cancer patients. Therefore, numerous clinical trials using CT antigen-derived immunogens have recently been undertaken.

The NY-ESO- 1 antigen is a classic CT antigen initially identified by SEREX (serological analysis of recombinant cDNA expression) analysis of an esophageal cancer patient and then found to be expressed in a variable proportion of a wide range of different tumor types, including melanoma, breast, ovary, and lung cancers (6). More recently, expression of NY-ESO-1 has also been reported in a high proportion $(80 \%)$ of synovial sarcomas $(7,8)$. Interestingly, at variance with a more heterogeneous expression pattern often found in tumors of other histological types, NY-ESO-1 expression in synovial sarcoma is remarkably homogeneous (7). 
Both humoral and cellular spontaneous responses to NY-ESO-1 have been reported in a high proportion of patients with advanced NY-ESO-1-expressing tumors $(9,10)$, underlining the high spontaneous immunogenicity of this antigen. Three peptides corresponding to overlapping sequences spanning the 155-167 region of the protein (155-163 QLSLLMWIT, 157-165 SLLMWITQC, and 157-167 SLLMWITQCFL) were initially shown to be recognized by a tumor-reactive $\mathrm{CD}^{+}$ $T$ cell line from a melanoma patient in a HLAA*0201-restricted (A2-restricted) fashion (10). By using a NY-ESO-1-specific tumor-reactive $\mathrm{CD}^{+} \mathrm{T}$ cell clone derived from tumor-infiltrating lymphocytes of a NY-ESO-1-expressing and seropositive patient, we have shown that, among these peptides, peptide 157-165 was the most efficiently recognized (11).

The immunogenicity of NY-ESO-1-derived peptides was first evaluated in a clinical trial of vaccination of cancer patients in which the three previously described peptides were administered intradermally, first alone and then in combination with GM-CSF (12), to cancer patients bearing NY-ESO-1-expressing progressive tumors. In NY-ESO-1 seronegative patients with no spontaneous $\mathrm{CD}^{+} \mathrm{T}$ cell response detectable before vaccination, a vigorous $\mathrm{CD}^{+} \mathrm{T}$ cell response to peptide 157-167 was observed, as detected by enzymelinked immunosorbent spot (ELISPOT), in four of seven vaccinated patients, whereas reactivity against peptide 157-165 appeared later and at a lower level. No reactivity to peptide $155-163$ was detected in any of the vaccinated patients.

In this study, we analyzed the $\mathrm{CD}^{+} \mathrm{T}$ cell response to a NY-ESO-1 peptide vaccine in two synovial sarcoma patients and one leiomyosarcoma patient bearing NY-ESO-1-expressing lesions. Because of the data reported previously (12), we chose to administrate a peptide vaccine containing peptides 157-165 and 157-167 together with GM-CSF. By analyzing the response to this vaccine with A2/peptide fluorescent multimeric complexes (multimers) incorporating overlapping A2 binding peptides in the 157-167 region, we detected a heterogeneous $\mathrm{CD}^{+}$ $T$ cell response directed against several distinct overlapping epitopes contained in this region. However, among the multiepitope-directed $\mathrm{CD}^{+} \mathrm{T}$ cells elicited by the vaccine, only those recognizing peptide $157-165$ with sufficiently high functional avidity recognized the naturally processed target on NY-ESO- $1^{+}$tumor cells, whereas other overlapping epitopes did not appear to be significantly expressed by NY-ESO- $1^{+}$tumor cells.

\section{Methods}

Patients and vaccination protocol. Response to vaccination with NY-ESO-1 peptides was analyzed in two synovial sarcoma patients (LUD 24/1 and LUD 24/3) and one leiomyosarcoma patient (LUD 24/2). The three patients expressed A2 and had NY-ESO-1-expressing lesions. Informed consent was obtained for enrollment in an immunization protocol sponsored by the Ludwig Institute for Cancer Research (LUD 00-024). Patients were immunized at 2-week intervals intradermally with $100 \mu \mathrm{g}$ of peptides NY-ESO-1 157-165 and 157-167 each in $300 \mu \mathrm{l} 100 \%$ DMSO at separate sites of the forearm, for 11 weeks. GM-CSF $(100 \mu \mathrm{g} /$ day $)$ was given subcutaneously as an adjuvant, daily, for 5 days, beginning 2 days before each set of peptide injections.

A2/peptide multimers and flow cytometry immunofluorescence analysis. PE-conjugated multimeric A2/peptide complexes containing NY-ESO-1-derived peptides (Table 1) were synthesized as described $(13,14)$. Samples were stained with A2/peptide multimers (4.5 $\mu \mathrm{g} / \mathrm{ml}$ ) in PBS containing $0.2 \%$ BSA for 1 hour at room temperature, washed once in the same buffer, stained with anti-CD8 mAb (Becton Dickinson Immunocytometry Systems, San Jose, California, USA) for $20 \mathrm{~min}$ utes at $4^{\circ} \mathrm{C}$, washed again, and analyzed by flow cytometry (Becton Dickinson Immunocytometry Systems). Data analysis was performed using Cell Quest software.

Cells. Patients' PBMCs were cultured in vitro for 1 week in CTL medium (15) containing hrIL-2 (10 IU $/ \mathrm{ml}$; Roche Pharma, Basel, Switzerland) and hrIL-7 (10 $\mathrm{ng} / \mathrm{ml}$; R\&D Systems Europe Ltd., Abingdon, United Kingdom) in the presence of either peptide $157-165$ or peptide $157-167$ ( $1 \mu \mathrm{M}$, added at day 1$)$. To reduce disulfide bonds present in the peptide solution, NY-ESO-1 peptides were preincubated for 1 hour at room temperature with the disulfide-reducing agent Tris [2-carboxyethyl] phosphine ([TCEP] $2 \mathrm{mM}$; Pierce Biotechnology, Rockford, Illinois, USA) (Weisan Chen, personal communication) before being used to stimulate patients' PBMCs or before functional assays (see below). In the latter case TCEP was further added during the assay at a final concentration of $500 \mu \mathrm{M}$. $\mathrm{A} 2 /$ peptide multimer $^{+} \mathrm{CD}^{+} \mathrm{T}$ cells were purified from PBMCs either ex vivo or after in vitro stimulation with the indicated peptide by flow cytometry cell sorting and cloned by limiting dilution culture in the presence of PHA (Sigma-Aldrich, St. Louis, Missouri, USA), allogeneic irradiated PBMCs, and hrIL-2 as described (15). Clones were subsequently expanded by periodic (3-4 weeks) restimulation under the same conditions.

A2 binding and antigen recognition assays. Antigen recognition was assessed by chromium release assay (CTL assay). The $\mathrm{A} 2^{+}$human mutant cell line CEMx721.T2 $\left(\mathrm{T} 2, \mathrm{~A}^{+} \mathrm{NY}-\mathrm{ESO}-1^{-}\right)(16)$ or the melanoma cell lines $\mathrm{Me}$ $275\left(\mathrm{~A} 2^{+} \mathrm{NY}-\mathrm{ESO}-1^{+}\right)$, NA8 (A2 $\left.{ }^{+} \mathrm{NY}-\mathrm{ESO}-1^{-}\right)$, Me 260 $\left(\mathrm{A} 2^{-}\right)$were used as targets. Briefly, after labeling with ${ }^{51} \mathrm{Cr}$ for 1 hour at $37^{\circ} \mathrm{C}$ followed by extensive washing, target cells $(1,000 /$ well) were incubated with effector cells at the indicated effector-to-target cell ratio $(\mathrm{E} / \mathrm{T})$ for 4 hours at $37^{\circ} \mathrm{C}$ in the absence or in the presence of the indicated synthetic peptide $(1 \mu \mathrm{M})$. In peptide titration experiments, target cells were incubated with effectors at an E/T ratio of 10:1 in the presence of serial dilutions of the indicated peptide. Chromium release was measured in the supernatant of the cultures using a $\gamma$-counter. The percentage of specific lysis was calculated as $100 \times[$ (experimental - spontaneous release $) /$ (total - spontaneous release)]. Peptide binding to A2 
Table 1

Binding capacity to HLA-A*0201 of NY-ESO- 1 peptides in the 157-167 region

$\begin{array}{ccc}\text { Peptide } \quad \text { Sequence } & \begin{array}{c}\text { Relative } \\ \text { predicted } \\ \text { binding }\end{array} & \begin{array}{c}\text { Relative } \\ \text { experimental } \\ \text { binding }\end{array}\end{array}$

NY-ESO-1 9-mers:

$\begin{array}{lccc}9-157 & \text { SLLMWITQC } & 1 & 1 \\ 9-158 & \text { LLMWITQCF } & 0.01 & 0.9 \\ 9-159 & \text { LMWITQCFL } & 28 & 6.8\end{array}$

NY-ESO-1 10-mers:

\begin{tabular}{lccc}
$10-157$ & SLLMWITQCF & 0.007 & 0.4 \\
$10-158$ & LLMWITQCFL & 17 & 1.2 \\
Flu-Ma 58-66 & GILGFVFTL & 13 & 35 \\
\hline
\end{tabular}

APredicted binding to HLA-A*0201 was determined using an algorithm that calculates the theoretical dissociation rate of the corresponding A2/peptide complexes (http://bimas.dcrt.nih.gov/molbio/hla-bind). Predicted binding is expressed relative to peptide NY-ESO-1 9-157. ${ }^{\text {BExperimental binding was }}$ assessed by functional competition assay as detailed in the Methods section. Experimental binding is expressed relative to peptide NY-ESO-1 9-157. Values are calculated as a mean of three independent experiments.

was assessed in a functional competition assay based on inhibition of recognition of the antigenic peptide tyrosinase $368-376$ by A2-restricted-specific CTL as described previously (11). IFN- $\gamma$ ELISPOT assay (17) was performed as described previously (11).

Degradation of NY-ESO-1 peptides in human serum. Peptides were added to human serum (preheated for 10 minutes at $37^{\circ} \mathrm{C}$ ) to a final concentration of $0.5 \times 10^{-4}$ $\mathrm{M}$ and incubated at $37^{\circ} \mathrm{C}$. Aliquots $(150 \mu \mathrm{l})$ were removed at the indicated time points and immediately frozen in liquid nitrogen to stop enzymatic reactions. Degradation samples were then tested in CTL peptide titration experiments on ${ }^{51} \mathrm{Cr}$-labeled T2 cells as described above. Peptide degradation experiments, for both NY-ESO-1 and Melan-A control peptides, were performed under reducing conditions using TCEP as detailed above.

\section{Results}

Detection of NY-ESO-1 157-165-specific immune responses in sarcoma patients vaccinated with NY-ESO-1 peptides 157-165 and 157-167. PBMC samples available from three sarcoma patients were stimulated in vitro for 1 week with either peptide NY-ESO-1 157-165 (9-157) or 157-167 (11-157). Cultures were initially monitored by staining with A2/peptide multimers (M) containing peptide 9-157 (M 9-157, hereafter). M 9-157 ${ }^{+} \mathrm{CD}^{+} \mathrm{T}$ cells were clearly detectable for patient LUD 24/1 in postvaccination but not in prevaccination samples and after stimulation with either peptide 9-157 or 11-157 (Figure 1). Similar results were obtained for patient LUD 24/3. For patient LUD $24 / 2$, no prevaccination sample was available; however, $M$ 9-157 ${ }^{+} \mathrm{CD} 8^{+} \mathrm{T}$ cells were detectable in the postvaccination sample after in vitro stimulation with peptide 11-

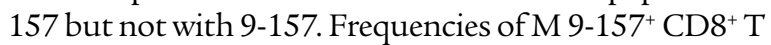
cells in in vitro peptide-stimulated postvaccination samples from the three patients are reported in Table 2.
Identification of additional potential A2-restricted epitopes in the NY-ESO-1 157-167 region. To more comprehensively assess the response to the vaccine, we inspected the 11-157 sequence to determine if the latter could contain, in addition to 9-157, other not previously described CTL epitopes. As summarized in Table 1, this region includes two additional nonapeptides and two decapeptides that may be presented in association with $\mathrm{A} 2$. The potential binding capacity to $\mathrm{A} 2$ of 9 - and 10-mers in the 157-167 region was first determined using an algorithm that calculates the theoretical dissociation rate of the corresponding A2/peptide complexes based on their amino acid composition (available at Bioinformatics and Molecular Analysis section of the NIH: http://bimas.dcrt.nih.gov/molbio/ hla-bind; ref. 18). Experimental binding was assessed by using a functional competition assay as described previously (11). Peptides 159-167 (9-159) and 158-167 (10-158) had a predicted binding superior to 9-157. This was confirmed by the experimental data in the case of 9-159 (Table 1), whereas for 10-158 experimental binding was comparable to 9-157. In contrast, peptides 158-166 (9-158) and 157-166 (10-157) had very poor predicted binding. However, their experimental binding was comparable to that of 9-157. In support of the experimental binding data, efficient refolding of $\mathrm{A} 2$ /peptide multimers was obtained for all NY-ESO-1 peptides listed in Table 1.

Sarcoma patients vaccinated with NY-ESO-1 peptides 9-157 and 11-157 respond to distinct overlapping A2-restricted epitopes in the 157-167 region. Postvaccination peptide-stimulated PBMC cultures from the three patients previously stained with M 9-157 (Figure 1 and Table 2) were stained with the panel of A2/peptide multimers incorporating each of the 9-and 10-mers in Table 1 . As summarized in Table 2, no significant staining was obtained in samples stimulated with 9-157 with any A2/ NY-ESO-1 multimers other than the one incorporating

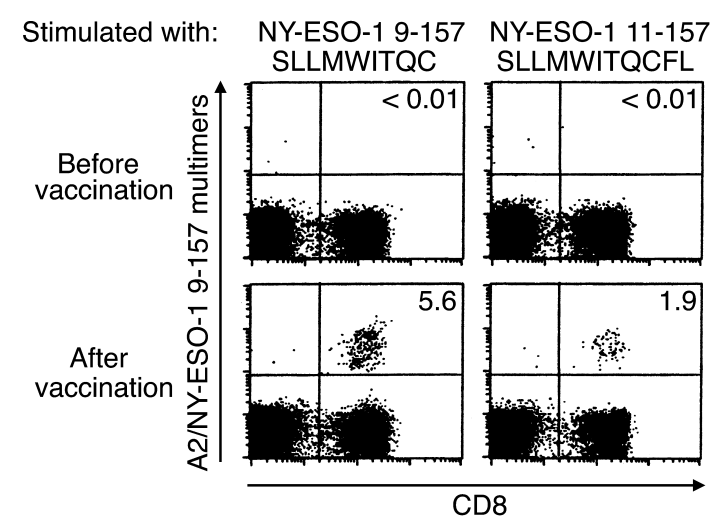

Figure 1

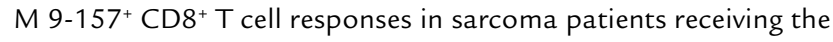
NY-ESO-1 peptide vaccine. Before and after vaccination PBMC samples from patient LUD 24/1 (after-vaccination sample day 169) were stimulated in vitro with peptide NY-ESO-1 9-157 or with peptide NY-ESO-1 11-157 (1 $\mu \mathrm{M})$, cultured during 1 week, and stained

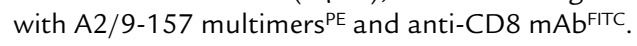


Table 2

Detection of specific $\mathrm{CD} 8^{+}$populations by staining of in vitro-stimulated PBMCs with the corresponding A2/peptide multimers

\begin{tabular}{|c|c|c|c|c|c|c|}
\hline \multirow{2}{*}{$\begin{array}{l}\text { Patient } \\
\text { Stimulated with: } \\
\text { Stained with: }\end{array}$} & \multicolumn{2}{|c|}{ LUD $24 / 1^{\mathrm{A}}$} & \multicolumn{2}{|c|}{ LUD $24 / 2$} & \multicolumn{2}{|c|}{ LUD $24 / 3$} \\
\hline & $9-157$ & $11-157$ & $9-157$ & $11-157$ & $9-157$ & $11-157$ \\
\hline M 9-157 & $5.6^{\mathrm{B}}$ & 1.9 & $<0.01$ & 0.5 & 0.07 & 0.06 \\
\hline M 9-158 & $<0.01$ & 0.19 & $<0.01$ & $<0.01$ & $<0.01$ & $<0.01$ \\
\hline M 9-159 & $<0.01$ & 10.0 & $<0.01$ & 6.5 & $<0.01$ & 5.3 \\
\hline M 10-157 & 0.27 & $<0.01$ & $<0.01$ & $<0.01$ & $<0.01$ & $<0.01$ \\
\hline M 10-158 & $<0.01$ & 5.7 & $<0.01$ & 0.12 & $<0.01$ & 0.14 \\
\hline
\end{tabular}

APostvaccination samples were from day 169,70 , and 102 for patients LUD 24/1, LUD 24/2, and LUD $24 / 3$, respectively. ${ }^{B}$ Values represent percentage of A2/peptide multimer ${ }^{+}$cells among $\mathrm{CD} 8^{+} \mathrm{T}$ cells. Significant values are shown in bold. $\mathrm{M}, \mathrm{A} 2 /$ peptide multimers.

9-157. The only exception was a relatively small population of $\mathrm{M} 10-157^{+} \mathrm{CD}^{+} \mathrm{T}$ cells $(0.27 \%$; Table 2$)$ seen in patient LUD 24/1. In contrast, in cultures stimulated with peptide 11-157, A2/peptide multimer ${ }^{+} \mathrm{T}$ cells were detected with multimers incorporating several other NY-ESO-1 peptides. First, a significant proportion of $M$ 9-159+ $\mathrm{CD}^{+} \mathrm{T}$ cells were detectable for all patients. Interestingly, $\mathrm{CD}^{+} \mathrm{T}$ cell responses to this epitope were found in a cancer patient following a similar vaccination protocol in a parallel trial (sponsored by the Ludwig Institute for Cancer Research and ongoing at the Weill Medical College of Cornell University, New York, New York, USA; Sacha Gnjatic, personal communication). Second, a significant proportion of $\mathrm{M} 10-158^{+}$ $\mathrm{CD}^{+} \mathrm{T}$ cells were detected for patient LUD 24/1 and to a lesser extent for patients LUD 24/2 and LUD 24/3. Finally, a small proportion of $\mathrm{M} 9-158^{+} \mathrm{CD} 8^{+} \mathrm{T}$ cells were detected in the case of patient LUD 24/1 only. Several of the above populations were isolated from the corresponding culture by multimer guided cell sorting and further expanded in vitro by stimulation in the presence of allogeneic irradiated feeder cells and PHA as described previously (15). The isolated populations were uniformly stained by the autologous A2/peptide multimers used for cell sorting. However, remarkably, none of these populations were significantly cross-stained by multimers incorporating the other NY-ESO-1-derived peptides (shown in Figure 2 for representative populations). Therefore, three distinct types of polyclonal populations, each specifically stained by the autologous A2/NY-ESO-1 peptide multimers used to isolate them (M 9-157, M 9-159, and M 10-158), were identified.

Fine specificity of antigen recognition of distinct NY-ESO-1 multimer ${ }^{+} C D 8^{+} T$ cells recognizing overlapping epitopes in the 157-167 region. The fine specificity of antigen recognition of the CTL populations identified by multimers incorporating the different NY-ESO-1 peptides, as well as their extent of cross-recognition, was first analyzed in an ELISPOT assay (Table 3). $\mathrm{CD}^{+} \mathrm{T}$ cells isolated with M 9-157 crossrecognized 10-157 only; those isolated with M 9-159 cross-recognized 10-158 only; and those isolated with M 10-158 cross-recognized 9-159 and, in one case, 9-158. The relative efficiency of peptide cross-recognition was further assessed in peptide titration experiments in a functional CTL assay (results are shown, for representative populations, in Figure 3a). Each of the three population types, characterized by a distinct pattern of multimer staining (Figure 2), recognized the peptide incorporated in multimers used for their isolation more efficiently than the other peptides. Significant cross-recognition of some of the latter, however, was found in each case. In line with the ELISPOT data, $\mathrm{CD}^{+} \mathrm{T}$ cells isolated with M 9-157 cross-recognized 10-157 more efficiently than the other peptides; those isolated with M 9-159 more efficiently cross-recognized 10-158; and those isolated with M 10-158 more efficiently cross-recognized 9-159. It is noteworthy that all sorted populations recognized peptide 11-157 with a roughly comparable efficiency. Polyclonal populations isolated from different individuals with multimers incorporating a given peptide exhibited similar cross-recognition patterns. In addition, similar results, in terms of cross-recognition patterns, were obtained with monoclonal populations

\section{Figure 2}

Sarcoma patients vaccinated with NY-ESO-1 peptides 157-165 and 157-167 respond to distinct overlapping A2-restricted epitopes in the 157-167 region. Polyclonal monospecific $\mathrm{CD}^{+}{ }^{+}$multimer ${ }^{+}$populations were isolated by cell sorting with the corresponding multimers and further expanded in vitro by mitogen-driven stimulation. After 2 weeks of in vitro expansion, each population was stained with multimers incorporating each of the overlapping 9-mer and 10-mer in the 157-167 region.

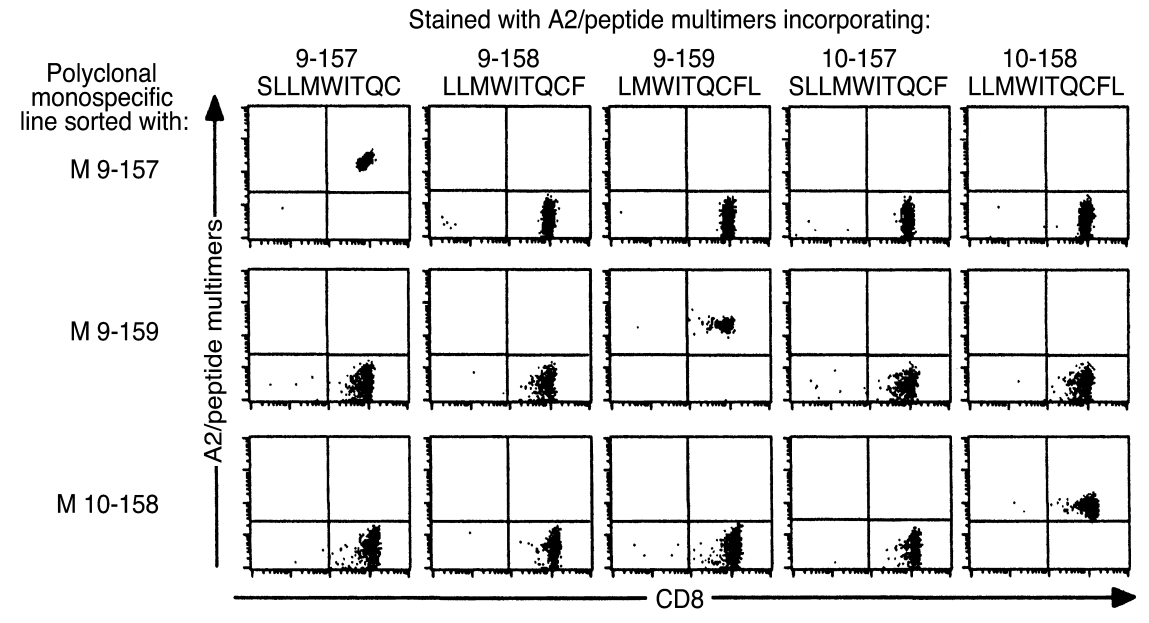


derived from each of these cultures, although the efficiency of cross-recognition varied to some extent among clones (not shown).

Tumor reactivity of NY-ESO-1 multimer ${ }^{+} \mathrm{CD} 8^{+} \mathrm{T}$ cells recognizing overlapping epitopes in the 157-167 region. The ability of the different A2/NY-ESO-1 peptide multimer isolated populations to specifically recognize NY-ESO-1expressing tumor cells was assessed by both ELISPOT (summarized in Table 3) and CTL assay (Figure 3b). As compared with the tumor-reactive CTL clone LAU $156 / 5$ (19) used as an internal control, no significant tumor recognition was detected in ELISPOT experiments as determined by analyzing 400 cells/well. However, when analyzing 4,000 cells/well, specific recognition of NY-ESO-1-expressing tumor cells was clearly detectable with M 9-157 sorted cells only. The number of spots obtained was, however, much lower than that obtained with the control clone LAU 156/5. Similar results were obtained in CTL assay; i.e., a low but specific level of lysis of NY-ESO-1-expressing tumor cells, was obtained for M 9-157 sorted cells but not for M 9-159 or M 10-158 sorted populations. To clarify the reason for the low level of tumor recognition obtained with the $M$ 9-157 polyclonal specific population as compared with the control clone LAU $156 / 5$, we analyzed single clones obtained from $\mathrm{M} 9-157^{+}$ $\mathrm{CD}^{+}$cells by limiting dilution culture as described previously (20). We found both tumor-reactive (i.e., clone LUD 24/1 1E5) and nontumor-reactive clones (i.e., clone LUD 24/1 3A7) (Figure 4a). Consistent with our previous data showing that functional avidity of antigen recognition often correlates with tumor recognition, tumor-reactive clones recognized 9-157 more efficiently than nontumor-reactive clones. However, as reported previously (21), the difference in functional avidity of peptide recognition between tumor-reactive and nontumor-reactive clones was relatively small in this antigenic system (less than tenfold difference in the 9-157 peptide dose required to achieve $50 \%$ maximal lysis; Figure $4 \mathrm{~b}$ ). Therefore, the low level of tumor recognition obtained with the $\mathrm{M}$ $9-157^{+} \mathrm{CD}^{+}$polyclonal population was explained by a functional heterogeneity of the composing clones, only part of which was able to recognize antigen-expressing tumor cells. It is of note that, similar to what was obtained

Table 3

LUD 24/1

LUD 24/2

LUD $24 / 3$

Clone LAU 156/5

LUD 24/1

LUD 24/1

LUD $24 / 2$

LUD $24 / 3$ with polyclonal monospecific populations, clones derived from either M 9-159- or M 10-158-sorted T cells failed to recognize NY-ESO-1-expressing tumors, despite their high functional avidity of antigen recognition (Figure 3).

Ex vivo analysis of vaccine-induced 9-157- and 9-159- specific $C D 8^{+} T$ cells. To further investigate if the results obtained after in vitro stimulation reflected the in vivo status in vaccinated patients, we performed the following experiments. First, the relative frequency of M 9-157 and $\mathrm{M}$ 9-159 $\mathrm{CD}^{+} \mathrm{T}$ cells was directly assessed ex vivo in PBMCs from patients LUD 24/2 and LUD 24/3. As shown in Figure 5a, M 9-159 CD8 ${ }^{+} \mathrm{T}$ cells were clearly detectable ex vivo in the case of patient LUD 24/3 (at a frequency of about $1 / 8,000 \mathrm{CD}^{+} \mathrm{T}$ cells), whereas the frequency of $\mathrm{M}$ 9-157 $\mathrm{CD}^{+} \mathrm{T}$ cells was, for this patient, below $1 / 50,000 \mathrm{CD}^{+} \mathrm{T}$ cells. In the case of circulating lymphocytes from patient LUD 24/2, both M 9-157 and M 9-159 CD8 ${ }^{+} \mathrm{T}$ cells were detectable ex vivo, the frequency of the latter population being about twofold higher than that of the former $(1 / 13,000$ and $1 / 6,000$ $\mathrm{CD}^{+} \mathrm{T}$ cells, respectively). To establish if functional

ELISPOT analysis of A2/NY-ESO-1 peptide multimer ${ }^{+}$populations

Peptide recognition

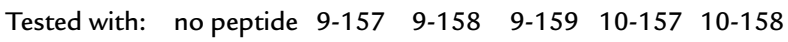

Stimulated with 9-157 Sorted with:

$\begin{array}{lllllll}\text { M 9-157 } & 0 & 77^{\mathrm{A}} & 0 & 0 & 24 & 0\end{array}$

Stimulated with 11-157 Sorted with:

M 9-157

M 9-159

M 10-158

0
0
0

M 9-159

0

M 9-159

M 10-158

0
0
0
NA8
400 cells

$\begin{array}{ccccc}0 & 0 & \mathbf{5 9} & 0 & \mathbf{5 6} \\ 0 & 0 & \mathbf{7 1} & 0 & \mathbf{4 2} \\ 0 & 19 & \mathbf{4 5} & 0 & \mathbf{5 1}\end{array}$

Tumor recognition

Tested with:

0

Me275
400 cells
NA8
4,000 cells Me275

Stimulated with 9-157:

Sorted with:

M 9-157

Stimulated with 11-157: Sorted with

M 9-157

M 9-159

M 10-158

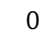

73

0

$>500$

M 9-159

M 9-159

M 10-158

0

$$
4
$$

0

46

94

0

0

0

0
0

0
0

0

0

0

0

0

0

0

0

0

AMean number of spots for duplicate wells; 400 cells/well were analyzed in each case for peptide recognition, whereas for tumor recognition either 400 or 4,000 cells/well were analyzed as indicated. Significant values are shown in bold. Antigen-presenting cells were T2 cells alone or in the presence of the indicated peptide for peptide recognition or the indicated tumor cell line (NA8, A2+ NY-ESO-1- or Me 275, $\mathrm{A}^{+}{ }^{\mathrm{NY}}-\mathrm{ESO}-1^{+}$) for tumor recognition. 

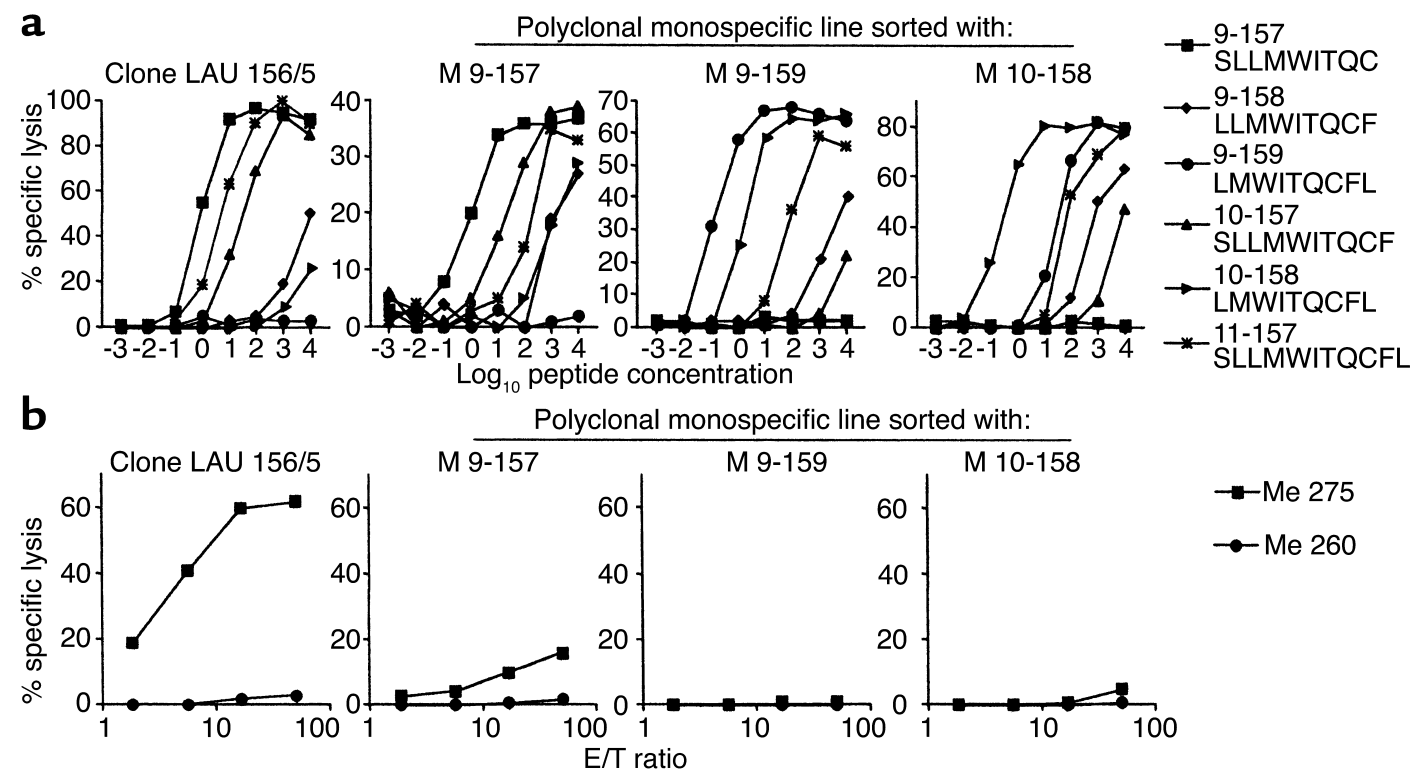

\section{Figure 3}

Fine specificity of antigen recognition and tumor reactivity of distinct NY-ESO-1 multimer ${ }^{+}$CTL recognizing overlapping epitopes in the 157-167 region. (a) Recognition of NY-ESO-1 peptides by polyclonal monospecific multimer+-sorted populations as well as by the NY-ESO-1-specific tumor-reactive clone LAU $156 / 5$ used as an internal control was assessed in a ${ }^{51} \mathrm{Cr}$ release assay on T2 target cells in the presence of graded concentration of the indicated peptides. (b) Tumor recognition was similarly assessed on Me $275\left(\mathrm{~A} 2^{+} \mathrm{NY}-\mathrm{ESO}-1^{+}\right)$or Me $260\left(\mathrm{~A} 2^{-}\right)$tumor target cells at the indicated $\mathrm{E} / \mathrm{T}$ ratio.

heterogeneity of antigen recognition and tumor reactivity of $\mathrm{M}$ 9-157 $\mathrm{CD}^{+} \mathrm{T}$ cells was also found ex vivo, $\mathrm{M}$ 9-157 CD8 ${ }^{+} \mathrm{T}$ cells were isolated from PBMCs of patient LUD 24/1 (the ex vivo frequency of the M 9-157 multi$\mathrm{mer}^{+} \mathrm{CD}^{+} \mathrm{T}$ cell population in this patient was about $1 / 10,000 \mathrm{CD}^{+} \mathrm{T}$ cells; Figure $5 \mathrm{~b}$ ) by direct ex vivo sorting and cloned under limiting dilution conditions. As illustrated in Figure 5b, four of five specific 9-157 CD8 ${ }^{+}$ $\mathrm{T}$ cell clones isolated following this procedure were able to specifically lyse NY-ESO-1-expressing tumor targets only in the presence of exogeneously added peptide, while the fifth was tumor reactive in the absence of peptide. Taken together, these data clearly show that the two main findings of this study, meaning the presence of substantial vaccine-elicited $\mathrm{CD}^{+} \mathrm{T}$ cell responses directed against cryptic epitopes and the poor tumor reactivity of most vaccine-elicited 9-157-specific $\mathrm{CD}^{+}$ $\mathrm{T}$ cells, were found when analyzing vaccine-induced $\mathrm{CD}^{+} \mathrm{T}$ cell responses ex vivo, similar to what was obtained with peptide-stimulated cultures.

NY-ESO-1 peptides in the 157-167 region exbibit a remarkable resistance to degradation by serum proteases. It was clear from both previously reported data (12)

\section{Figure 4}

Functional clonal heterogeneity in M 9-157 polyclonal population's impact on tumor reactivity. (a) Tumor recognition by $T$ cell clones

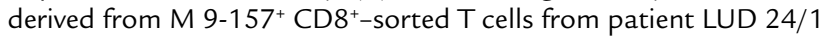
was assessed on Me275 (A2 ${ }^{+} \mathrm{NY}-\mathrm{ESO}-1^{+}$) or NA8 (A2 ${ }^{+} \mathrm{NY}-\mathrm{ESO}-1^{-}$) tumor target cells at the indicated $\mathrm{E} / \mathrm{T}$ ratio. (b) Functional avidity

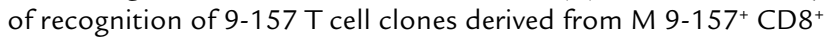
sorted T cells from patient LUD $24 / 1$ as well as by clone LAU $156 / 5$ used as an internal control was assessed on T2 target cells in the presence of graded peptide concentrations. and the data reported above, that, compared with most other tumor antigen-derived peptides (22), NY-ESO-1 peptides used in this study, and particularly peptide 157-167, exhibited an uncommon immunogenicity. Although this can partially be ascribed to the presence within this region of multiple overlapping $\mathrm{A} 2$-restricted $\mathrm{CD} 8^{+} \mathrm{T}$ cell epitopes, as reported above, other factors such as susceptibility to degradation by serum proteases have been shown to

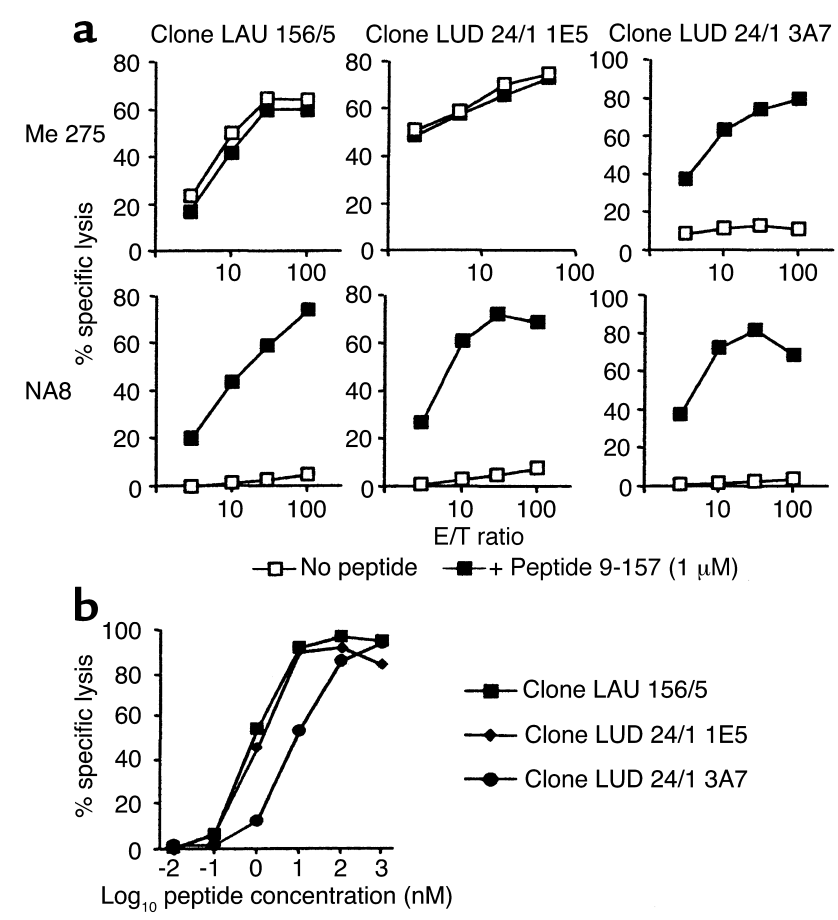




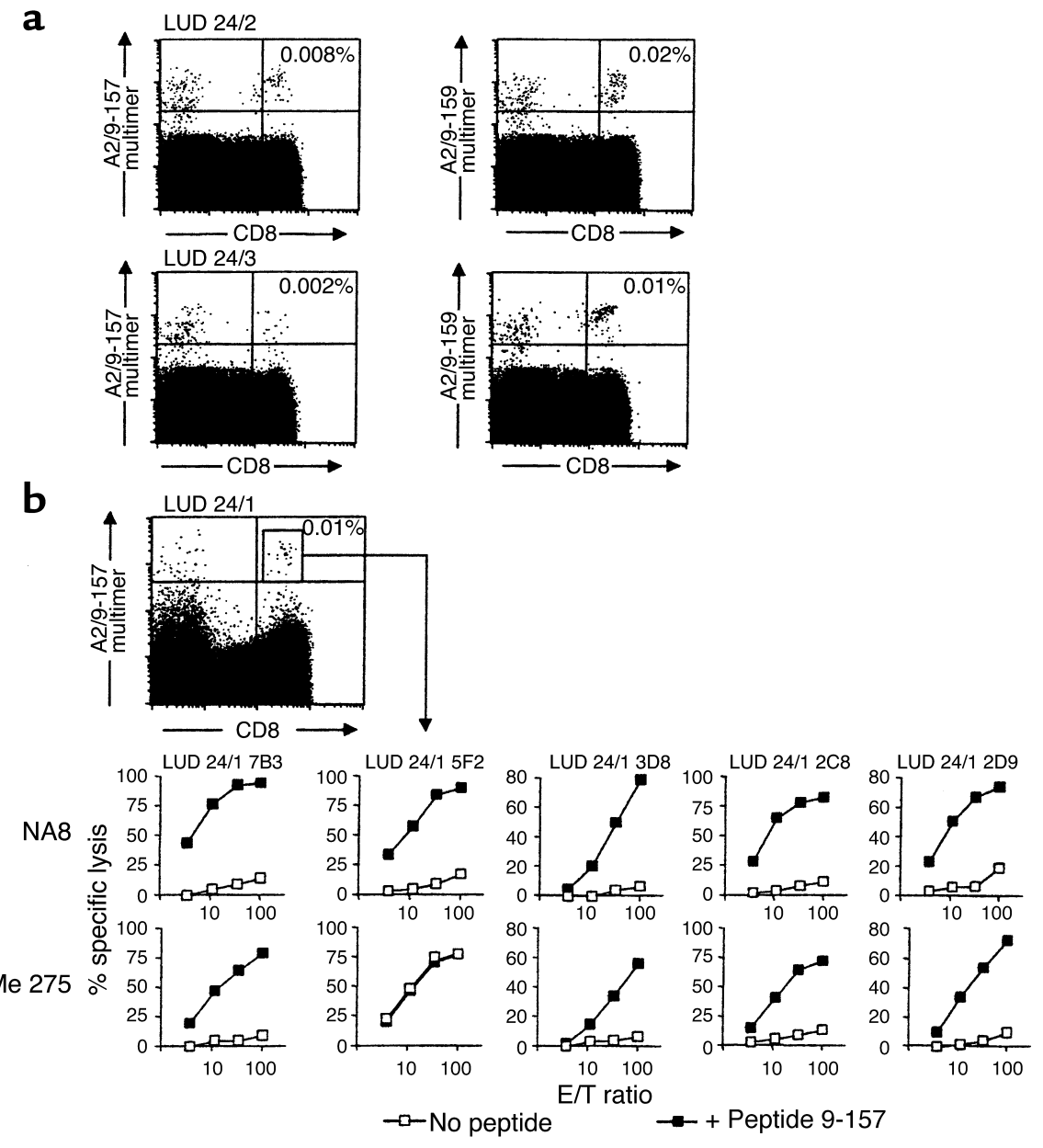

\section{Figure 5}

Ex vivo analysis of vaccine-induced 9-157and 9-159-specific CD8 ${ }^{+} \mathrm{T}$ cells. (a) Cryopreserved PBMCs from vaccinated patients were thawed, incubated overnight at $37^{\circ} \mathrm{C}$ in $\mathrm{CTL}$ medium, and stained with $\mathrm{A} 2 / 9-157$ or $\mathrm{A} 2 / 9-159$ multimers $^{\mathrm{PE}}$ and anti-CD8 mAb ${ }^{\mathrm{FITC}}$. (b) PBMCs from patient LUD 24/1 were stained as in $\mathbf{a} . \mathrm{M}^{+} \mathrm{CD} 8^{+}$positive cells were directly isolated by cell sorting and cloned under limiting dilution conditions. Tumor recognition by $T$ cell clones obtained following this procedure was assessed on Me275 $\left(\mathrm{A}^{+}{ }^{+} \mathrm{NY}-\mathrm{ESO}-1^{+}\right)$or NA8 $\left(\mathrm{A}^{+}\right.$ $\left.\mathrm{NY}-\mathrm{ESO}-1^{-}\right)$tumor target cells at the indicated $\mathrm{E} / \mathrm{T}$ ratio in the absence or in the presence of the corresponding peptide. greatly influence peptide immunogenicity (23). Therefore, it was interesting to analyze the degradation kinetics of NY-ESO-1 peptides in the 157-167 region in human serum. As illustrated in Figure 6 this analysis revealed a remarkable resistance to serum proteases of peptides 9-157 and 9-159, because no significant decrease in antigen recognition by the corresponding specific $\mathrm{T}$ cell population was detectable after 24-hour incubation in human serum (Figure 6a). Remarkably, similar results were obtained with peptide $11-157$ by using both M 9-157+ and $\mathrm{M} 9-159^{+}$populations (Figure $6 \mathrm{~b}$ ). Resistance to serum proteases was most likely related to the presence of $C$ at position 165, because a 9-157 peptide analogue bearing a single A substitution at this position was susceptible to degradation by serum proteases, similar to what we have previously reported for a Melan-A-derived peptide (Melan-A 27-35) (23) used here as an internal control (Figure 6c). Because of its resistance to serum proteases, we hypothesized that peptide 11-157 could bind to the A2 molecule and be presented to $\mathrm{CD}^{+} \mathrm{T}$ cells as such. Consistent with this hypothesis, efficient refolding of A2/11-157 multimers was obtained. Staining with M 11-157 of postvaccination samples stimulated with 11-157 revealed distinct populations of $\mathrm{M} 11-157^{+}$cells that accounted for $3.5,3.4$, and $0.47 \%$ of total $\mathrm{CD}^{+} \mathrm{T}$ cells in the cultures for patients LUD 24/1, LUD 24/2, and LUD 24/3, respectively. In contrast, no significant levels of $\mathrm{M} 11-157^{+} \mathrm{CD}^{+} \mathrm{T}$ cells were detected in cultures stimulated with $9-157$. M $11-157^{+} \mathrm{CD}^{+} \mathrm{T}$ cell populations were distinct from the ones detected with multimers incorporating shorter NY-ESO-1 peptides (Figure 2), because the latter were not crossstained by M 11-157 (not shown).

\section{Discussion}

Great attention is currently focused on the development of tumor vaccines that incorporate defined tumor antigen-derived peptides. In this regard, critical questions have emerged with respect to the optimal composition of such vaccines, in terms of the choice of the appropriate peptides, formulation (adjuvant and delivery mode), and molecular monitoring of the induced immune responses. In this study, we performed a thorough analysis of the $\mathrm{CD}^{+} \mathrm{T}$ cell response to a peptide vaccine composed of the NY-ESO-1 9-mer 157-165 and the 11-mer 157-167 by using A2/peptide multimers incorporating all potential 9-mers and 10-mers contained in the 11-mer.

A salient finding was the large heterogeneity of the response elicited by the vaccine that encompassed $T$ 

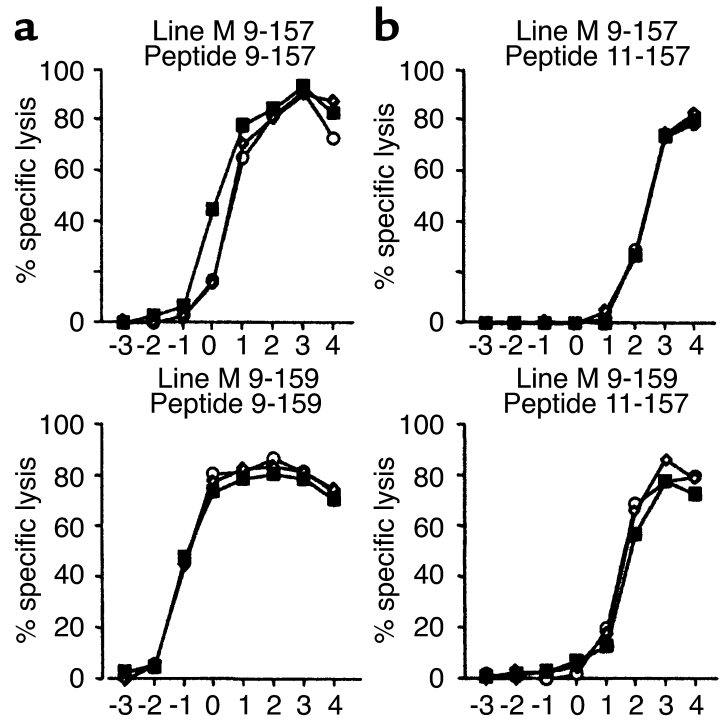

$\log _{10}$ peptide concentration $(\mathrm{nM})$

$\rightarrow$ Time $0 \rightarrow 2 \mathrm{~h}$

$\multimap 24 \mathrm{~h}$

\section{Figure 6}

Resistance of NY-ESO-1 peptides in the 157-167 region to degradation in human serum. NY-ESO-1 peptides 9-157, 9-157 substituted analogue C165A, 9-159, 11-157, or Melan-A 27-35 (AAGIGILTV) were incubated in human serum for the indicated time periods, and antigenic activity was then evaluated in CTL assay by using as effector cells the indicated NY-ESO-1 polyclonal monospecific lines or, in the case of the Melan-A peptide, the Melan-A-specific clone LAU 203/17. Antigen-specific lysis was assessed on ${ }^{51} \mathrm{Cr}$-labeled T2 cells in the presence of serial dilutions of each degradation sample. (a) Peptide 9-157 tested with line M 9-157 (upper panel) and peptide 9-159 tested with line M 9-159 (lower panel). (b) Peptide 11-157 tested with line M 9-157 (upper panel) or with line M 9-159 (lower panel). (c) Peptide 9-157 C165A tested with line M 9-157 (upper panel) and peptide Melan- $A_{27-35}$ tested with clone LAU 203/17 (lower panel). The human serum used in this experiment was heat-inactivated, but similar results were obtained by using fresh serum.

cells recognizing several overlapping epitopes in the 157-167 region. We isolated three types of $\mathrm{CD}^{+} \mathrm{T}$ cell populations that were clearly distinguishable by their pattern of multimer staining and their different fine specificity of recognition of NY-ESO-1 peptides. Interestingly, the populations identified by multimers incorporating 9-159 after in vitro stimulation of patients' PBMCs with peptide 157-167 were larger than that of most of the other populations, suggesting that $\mathrm{CD}^{+} \mathrm{T}$ cells specific for this peptide may dominate the overall response to the vaccine. Importantly, ex vivo staining of patients' PBMCs confirmed that $\mathrm{M} 9-159^{+} \mathrm{CD}^{+} \mathrm{T}$ cells could be detected among circulating lymphocytes at a frequency comparable or superior to that of $\mathrm{M} 9-157^{+} \mathrm{CD}^{+} \mathrm{T}$ cells. It is noteworthy that two additional populations were identified for patient LUD 24/1 upon staining with M 9-158 and M 10-157 (see Table 2). In addition, peptide 11-157 was able to bind to the A2 molecule and be recognized by distinct $\mathrm{CD}^{+} \mathrm{T}$ cell populations as such. Thus, taken together, the diversity of the response elicited by peptide 11-157 and detectable with multimers incorporating NY-ESO-1 peptides included six overlapping epitopes distinguishable by $\mathrm{CD} 8^{+} \mathrm{T}$ cells.
The molecular basis for the elicitation of this multiepitope response was elucidated by the finding that peptide $11-157$ is remarkably resistant to degradation by serum proteases and can bind to the A2 molecule as such. This is consistent with previous data indicating that, although most peptides naturally bound to the A2 molecule are nine amino acids long (24), longer peptides can also bind and be recognized by $\mathrm{CD}^{+} \mathrm{T}$ cells as distinct epitopes $(25,26)$. Peptide 11-157 contains three amino acids, L158, L159, and M160 in the amino-terminal part of the peptide, and L167 at the carboxyl-terminal end, that are major anchors for binding the A2 molecule. Therefore, this peptide can most likely bind to and be presented in association with the A2 molecule in various conformations. Our data show that A2/peptide multimers incorporating overlapping 9-and 10 -mers in the $157-167$ region can efficiently mimic, when binding the A2 molecule, some of these conformations that are distinguishable by $\mathrm{CD}^{+} \mathrm{T}$ cells. These results also reveal that $\mathrm{CD}^{+} \mathrm{T}$ cells able to recognize each of these different conformations are available in the $\mathrm{CD} 8^{+} \mathrm{T}$ cell repertoire of cancer patients and can be primed by the vaccine.

A multiepitope $\mathrm{CD}^{+} \mathrm{T}$ cell response to a tumor antigen-de-rived peptide vaccine is highly desirable if all or most of the composing $\mathrm{CD}^{+} \mathrm{T}$ cell specificities recognize epitopes that are efficiently processed and presented by tumor cells. Peptide 9-157 corresponds to the epitope that we have shown previously to be optimally recognized by tumor-reactive $\mathrm{CD}^{+} \mathrm{T}$ cells isolated from melanoma patients with spontaneous responses to NY-ESO-1 (11). Nonetheless, M 9-157+ $\mathrm{CD}^{+} \mathrm{T}$ cells isolated in this study from both in vitro peptide-stimulated PBMCs and, more importantly, directly from circulating lymphocytes, ex vivo, exhibited heterogeneous functional avidity of antigen recognition, and only a minority of them was reactive to NY-ESO-1-expressing tumors. Because of the simultaneous vaccination with peptide 9-157 and 11-157, it is not clear if one of these peptides, or both, is inducing the detected $9-157 \mathrm{CD}^{+} \mathrm{T}$ cell response. It is possible that high-avidity $9-157 \mathrm{CD}^{+} \mathrm{T}$ cells are exclusively induced by peptide 9-157. In favor of this hypothesis, we have recently observed a vigorous NY-ESO-1-specific tumor-reactive $\mathrm{CD}^{+} \mathrm{T}$ cell 
response in a melanoma patient vaccinated with peptide 9-157 alone in an independent trial (D. Valmori and D. Speiser, Ludwig Institute for Cancer Research, Lausanne Branch, unpublished data). Because of the ability of peptide 11-157 to bind to the A2 molecule in different conformations, A2/11-157 complexes may stimulate $\mathrm{M} 9-157^{+} \mathrm{T}$ cells that differ from those stimulated by A2/9-157 complexes in terms of fine specificity and therefore functional avidity of antigen recognition. However, as the vaccine was administered with different adjuvants in the two trials (Montanide was used as an adjuvant in the Lausanne trial), it remains possible that both tumor-reactive and nontumor-reactive cells could be induced by either or both 9-157 and 11-157 in the vaccine formulation used here.

It is of note that A2/NY-ESO- 1 multimer ${ }^{+} \mathrm{CD}^{+} \mathrm{T}$ cells specific for two of the additional epitopes identified here, including 9-159, were nontumor reactive. Interestingly, these results are in agreement with data obtained by using a proteasomal cleavage prediction program (developed at the Department of Biomathematics Immunology, the University of Tübingen, Tübingen, Germany; available at http://www.paproc. $\mathrm{de} /)(27,28)$ that predicts a cleavage site after residue 165 , but not after residue 167 . Therefore 9-159 and 10-158 correspond to "cryptic" epitopes that are presented after exposure to the antigen in peptide form, but not after endogenous proteasomal processing. It is of note that, in agreement with these results, no specific $\mathrm{CD}^{+} \mathrm{T}$ cell response to any epitope other than 9-157 was detected in samples from melanoma patients with spontaneous responses to NY-ESO-1 after in vitro stimulation with peptide 11-157 (not shown). Interestingly, in a recent study, the dominance of a peptide-MHC class II conformation that displays a cryptic $\mathrm{T}$ cell epitope in a myelin basic protein-derived 13-mer peptide accounted for the inability of the latter in modulating the course of experimental autoimmune encephalomyelitis (29). This suggests that the presence of dominant cryptic sequences within longer peptides can unpredictably influence their immunological properties.

Taken together, the data reported here demonstrate that vaccination with a synthetic NY-ESO-1 peptide that differs from the peptide optimally recognized by tumor-reactive $\mathrm{CD}^{+} \mathrm{T}$ cells only by the presence of two additional carboxyl-terminal natural amino acids can elicit a multiepitope $\mathrm{CD}^{+} \mathrm{T}$ cell response of unpredicted diversity that is mostly nonreactive to the tumor. These results raise concerns with respect to what might be an appropriate vaccine that efficiently and selectively stimulates tumor-reactive NY-ESO-1-specific CD8 ${ }^{+} \mathrm{T}$ cells. Because tumor recognition is selectively observed with high avidity 9-157-specific CD8 ${ }^{+} \mathrm{T}$ cells, this peptide should be used alone to stimulate $\mathrm{CD}^{+} \mathrm{T}$ cells in vivo. In general, when aiming at the elicitation of tumor-reactive $\mathrm{CD}^{+} \mathrm{T}$ cell responses, it would be recommended to use peptides that correspond as precisely as possible to defined $\mathrm{CD}^{+} \mathrm{T}$ cell epitopes naturally processed and expressed by tumor cells.

A NY-ESO-1 $T$ cell epitope that overlaps the 157-167 region and is recognized by $\mathrm{CD} 4^{+} \mathrm{T}$ cells in association with HLA-DP4 (an MHC class II allele commonly expressed in Caucasians) has been described recently (157-170) (30). CD4 ${ }^{+} \mathrm{T}$ cells specific for this epitope were isolated from the majority of NY-ESO-1-seropositive patients analyzed. In addition, and importantly, the large majority of melanoma patients with spontaneous NY-ESO-1specific $\mathrm{Ab}$ responses expressed the DP4 allele. Tumor antigen-specific CD $4^{+} \mathrm{T}$ cells play a central role in initiating and maintaining host immune responses against cancer (31). They exert their activity mainly by providing critical help for tumor-specific $\mathrm{CD}^{+} \mathrm{T}$ cells. It has been clearly shown that the immunogenicity of MHC class I peptides can be considerably augmented, both in terms of magnitude and life span of the elicited $\mathrm{CD}^{+} \mathrm{T}$ cell responses, by the simultaneous elicitation of $\mathrm{CD}^{+} \mathrm{T}$ cells, particularly if the latter are derived from the same antigen (32). In addition, tumor antigen-specific $\mathrm{CD}^{+} \mathrm{T}$ cells can provide help for tumor antigen-specific $\mathrm{Ab}$ production by B cells. Interestingly, accumulating experimental evidence indicates that tumor antigen-specific Ab's may play an important role in cancer immunity by promoting cross-presentation of MHC class I-restricted epitopes by dendritic cells (33).

In this context it is clear that the use of synthetic peptides encompassing both MHC-class I- and class II-restricted epitopes, as recently reported in the case of a HER-2/neu peptide vaccine (34), would be highly attractive because it could, in principle, result in the simultaneous elicitation of the different arms of specific immune responses to cancer. Nonetheless, the results of the present study indicate that this strategy may not be optimal in all cases and underline the importance of a thorough monitoring of the complete repertoire of specific $T$ cell responses elicited by such vaccines. A more "physiological" approach for the simultaneous elicitation of relevant NY-ESO-1-specific $\mathrm{CD}^{+} \mathrm{T}$ cell, $\mathrm{CD}^{+} \mathrm{T}$ cell, and $\mathrm{Ab}$ responses could consist in the administration of peptides corresponding to precisely defined $\mathrm{CD}^{+} \mathrm{T}$ cell epitopes in combination with recombinant NY-ESO-1 protein.

\section{Acknowledgments}

D. Valmori was partially supported by the Cancer Research Institute. We would like to thank Nicole Montandon and Mary Hesdorffer for technical assistance, Donata Rimoldi for kindly providing the tumor cell lines, and Pascal Batard for assistance in flow cytometry immunofluorescence cell sorting. C.S. Hesdorffer is supported by a grant from The Breast Cancer Alliance Inc. R.N. Taub is supported by gifts from Phillip L. Milstein to the Columbia Mesothelioma Center and from the Abraham and Phyllis Katz Foundation. 
1. Coulie, P.G., et al. 1994. A new gene coding for a differentiation antigen recognized by autologous cytolytic $\mathrm{T}$ lymphocytes on HLA-A2 melanomas. J. Exp. Med. 180:35-42.

2. Labrecque, S., Naor, N., Thomson, D., and Matlashewski, G. 1993. Analysis of the anti-p53 antibody response in cancer patients. Cancer Res. 53:3468-3471.

3. Cheever, M.A., et al. 1995. Immunity to oncogenic proteins. Immunol. Rev. 145:33-59.

4. Tindle, R.W. 1996. Human papillomavirus vaccines for cervical cancer. Curr. Opin. Immunol. 8:643-650.

5. van der Bruggen, P., et al. 1991. A gene encoding an antigen recognized by cytolytic $\mathrm{T}$ lymphocytes on a human melanoma. Science. 254:1643-1647.

6. Chen, Y.T., et al. 1997. A testicular antigen aberrantly expressed in human cancers detected by autologous antibody screening. Proc. Natl. Acad. Sci. USA. 94:1914-1918.

7. Jungbluth, A.A., et al. 2001. Monophasic and biphasic synovial sarcomas abundantly express cancer/testis antigen NY-ESO-1 but not MAGE-A1 or CT7. Int. J. Cancer. 94:252-256.

8. Jungbluth, A.A., et al. 2001. Immunohistochemical analysis of NY-ESO1 antigen expression in normal and malignant human tissues. Int. J. Cancer. 92:856-860.

9. Stockert, E., 1998. A survey of the humoral immune response of cancer patients to a panel of human tumor antigens. J. Exp. Med. 187:1349-1354.

10. Jager, E., et al. 1998. Simultaneous humoral and cellular immune response against cancer-testis antigen NY-ESO-1: definition of human histocompatibility leukocyte antigen (HLA)-A2-binding peptide epitopes. J. Exp. Med. 187:265-270.

11. Valmori, D., et al. 2000. Naturally occurring HLA-A2 restricted CD8+ T cell response to the cancer testis antigen NY-ESO-1 in melanoma patients. Cancer Res. 60:4499-4506.

12. Jager, E., et al. 2000. Induction of primary NY-ESO-1 immunity: CD8+ T lymphocyte and antibody responses in peptide-vaccinated patients with NY-ESO-1+ cancers. Proc. Natl. Acad. Sci. USA. 9:12198-12203.

13. Romero, P., et al. 1998. Ex vivo staining of metastatic lymph nodes by class I major histocompatibility complex tetramers reveals high numbers of antigen-experienced tumor-specific cytolytic $\mathrm{T}$ lymphocytes. J. Exp. Med. 188:1641-1650.

14. Altman, J.D., et al. 1996. Phenotypic analysis of antigen-specific T lymphocytes. Science. 274:94-96.

15. Valmori, D., et al. 1999. An antigen-targeted approach to adoptive transfer therapy of cancer. Cancer Res. 51:2167-2173.

16. Salter, R.D., Howell, D.N., and Cresswell, P. 1985. Genes regulating HLA class I antigen expression in T-B lymphoblast hybrids. Immunogenetics. 21:235-246.

17. Czerkinsky, C., et al. 1988. Reverse ELISPOT assay for clonal analysis of cytokine production. I. Enumeration of gamma-interferon-secreting cells. J. Immunol. Meth. 110:29-36.
18. Parker, K.C., Bednarek, M.A., and Coligan, J.E. 1994. Scheme for ranking potential HLA-A2 binding peptides based on independent binding of individual peptide side-chains. J. Immunol. 152:163-175.

19. Valmori, D., et al. 2000. Naturally occurring human lymphocyte antigenA2 restricted CD8 + T-cell response to the cancer testis antigen NY-ESO1 in melanoma patients. Cancer Res. 60:4499-4506.

20. Valmori, D., et al. 1998. Diversity of the fine specificity displayed by HLA-A*0201-restricted CTL specific for the immunodominant MelanA/MART-1 antigenic peptide [erratum 1999, 162:1093]. J. Immunol. 161:6956-6962.

21. Dutoit, V., et al. 2002. Functional avidity of tumor antigen-specific CTL recognition directly correlates with the stability of MHC/peptide multimer binding to TCR. J. Immunol. 168:1167-1171.

22. Melief, C.J., et al. 1996. Peptide-based cancer vaccines. Curr. Opin. Immunol. 8:651-657.

23. Blanchet, J.S., et al. 2001. A new generation of Melan-A/MART-1 peptides that fulfill both increased immunogenicity and high resistance to biodegradation: implication for molecular anti-melanoma immunotherapy. J. Immunol. 167:5852-5861.

24. Hunt, D.F., et al. 1992. Characterization of peptides bound to the class I MHC molecule HLA-A2.1 by mass spectrometry. Science. 255:1261-1263.

25. Romero, P., et al. 1997. Cytolytic T lymphocyte recognition of the immunodominant HLA-A*0201-restricted Melan-A/MART-1 antigenic peptide in melanoma. J. Immunol. 159:2366-2374.

26. Aarnoudse, C.A., van den Doel, P.B., Heemskerk, B., and Schrier, P.I 1999. Interleukin-2-induced, melanoma-specific $\mathrm{T}$ cells recognize CAMEL, an unexpected translation product of LAGE-1. Int. J. Cancer. 82:442-448.

27. Kuttler, C., et al. 2000. An algorithm for the prediction of proteasomal cleavages. J. Mol. Biol. 298:417-429.

28. Nussbaum, A.K., et al., 2001. PAProC: a prediction algorithm for proteasomal cleavages available on the WWW. Immunogenetics. 53:87-94.

29. Anderton, S.M., Viner, N.J., Matharu, P., Lowrey, P.A., and Wraith, D.C. 2002. Influence of a dominant cryptic epitope on autoimmune T cell tolerance. Nat. Immunol. 3:175-181.

30. Zeng, G., Wang, X., Robbins, P.F., Rosenberg, S.A., and Wang, R.F. 2002 CD4(+) T cell recognition of MHC class II-restricted epitopes from NYESO-1 presented by a prevalent HLA DP4 allele: association with NYESO- 1 antibody production. Proc. Natl. Acad. Sci. USA. 98:3964-3969.

31. Toes, R.E., Ossendorp, F., Offringa, R., and Melief, C.J. 1999. CD4 T cells and their role in antitumor immune responses. J. Exp. Med. 189:753-756.

32. Hung, K., et al. 1998. The central role of CD4(+) T cells in the antitumor immune response. J. Exp. Med. 188:2357-2368.

33. Rafiq, K., Bergtold, A., and Clynes, R. 2002. Immune complex-mediated antigen presentation induces tumor immunity. J. Clin. Invest. 110:71-79. doi:10.1172/JCI200215640.

34. Knutson, K.L., Schiffman, K., and Disis, M.L. 2001. Immunization with a HER-2/neu helper peptide vaccine generates HER-2/neu CD8 T-cell immunity in cancer patients. J. Clin. Invest. 107:477-484. 